\title{
Des petites localités fragiles dans une région en mal de développement : le cas du Bas-Saint-Laurent
}

\author{
$M$ ajella Simard ${ }^{1}$ \\ Université du Q uébec à Rimouski
}

\section{Introduction}

En octobre 1999, le Québec tout entier était sensibilisé aux graves problèmes de la Gaspésie. En effet, l'annonce de la fermeture de l'usine Gaspésia, à Chandler, associée aux difficultés de Mines et Exploration Noranda, à Murdochville, provoquait un spectaculaire raz-de-marée médiatique, tant du côté de la presse écrite qu'électronique. Si les vagues causées par ce flot d'informations n'inondent plus la une des journaux et ne font plus les manchettes du téléjournal, ce n'est pas parce que l'économie de la Gaspésie se porte mieux. Le sous-emploi affecte toujours de vastes parties de l'espace gaspésien en raison d'une structure économique qui repose essentiellement sur l'exploitation des ressources naturelles (essentiellement la pêche et la forêt) et le tourisme. Ces trois secteurs d'activité, qui procurent des emplois majoritairement saisonniers, souvent à temps partiel et faiblement rémunérés, sont en partie responsables du taux de chômage faramineux $(28 \%)$ que l'on retrouve au sein de cette région ${ }^{2}$. Les diverses mesures tampons mises de l'avant par les pouvoirs publics (l'implantation de deux centres d'appels téléphoniques, le maintien du lien ferroviaire entre Matapédia et Chandler et l'annonce d'une aide financière de 84 millions de dollars octroyée par les deux paliers de gouvernements pour encourager la diversification de l'économie) n'auront pas changé grand-chose aux profondes disparités socio-économiques qui se manifestent depuis plusieurs années en Gaspésie. Tout au plus elles auront permis, d'une part, de soulager l'hémorragie gaspésienne dont les causes semblent davantage attribuables à des facteurs d'ordre structurel qu'à une crise passagère et, $d$ ' autre part, de tempérer les ardeurs des Patriotes, un mouvement militant né en décembre 1999 à la suite de la fermeture de la Gaspésia ${ }^{3}$.

Bien que les problèmes de développement semblent particulièrement se concentrer à l'est de la province, ils ne sont pas moins présents dans d'autres régions du Québec, notamment dans celle du Bas-St-Laurent, qui partage plusieurs points communs avec son homologue gaspésienne. Décroissance démographique, taux de chômage élevé en raison de la saisonnalité et de la rareté des emplois, insuffisance de services, faiblesse des revenus, diminution du potentiel forestier sont autant d'éléments qui handicapent sérieusement l'économie bas-laurentienne. S'ajoutent à ces handicaps différentes caractéristiques socio-spatiales et géographiques liées notamment au potentiel biophysique et à la structure du peuplement (la vaste superficie du territoire, la dispersion de la population et des constructions, la forte dominance des espaces non bâtis, la présence de nombreuses localités de petite taille démographique, l'étirement du tissu de peuplement, la distance qui sépare les localités les unes des autres et l'absence d'un véritable pôle de développement capable d'impulsion économique), divers facteurs à caractère politico-économique (les lois et mécanismes de l'économie, les politiques gouvernementales, fiscales et monétaires, les aléas de la conjoncture nationale et internationale, etc.) ainsi que certaines considérations d'ordre symbolique ou perceptuel. Ces éléments, qui influencent à des degrés divers l'organisation socio-économique et l'avenir de nombreuses municipalités, contribuent à maintenir plusieurs parties du territoire bas-laurentien dans un état de mal-développement régional. C'est le cas notamment 


\section{Carte 1. Les petites et très petites localités rurales dans l'assiette socio-spatiale bas-laurentienne}

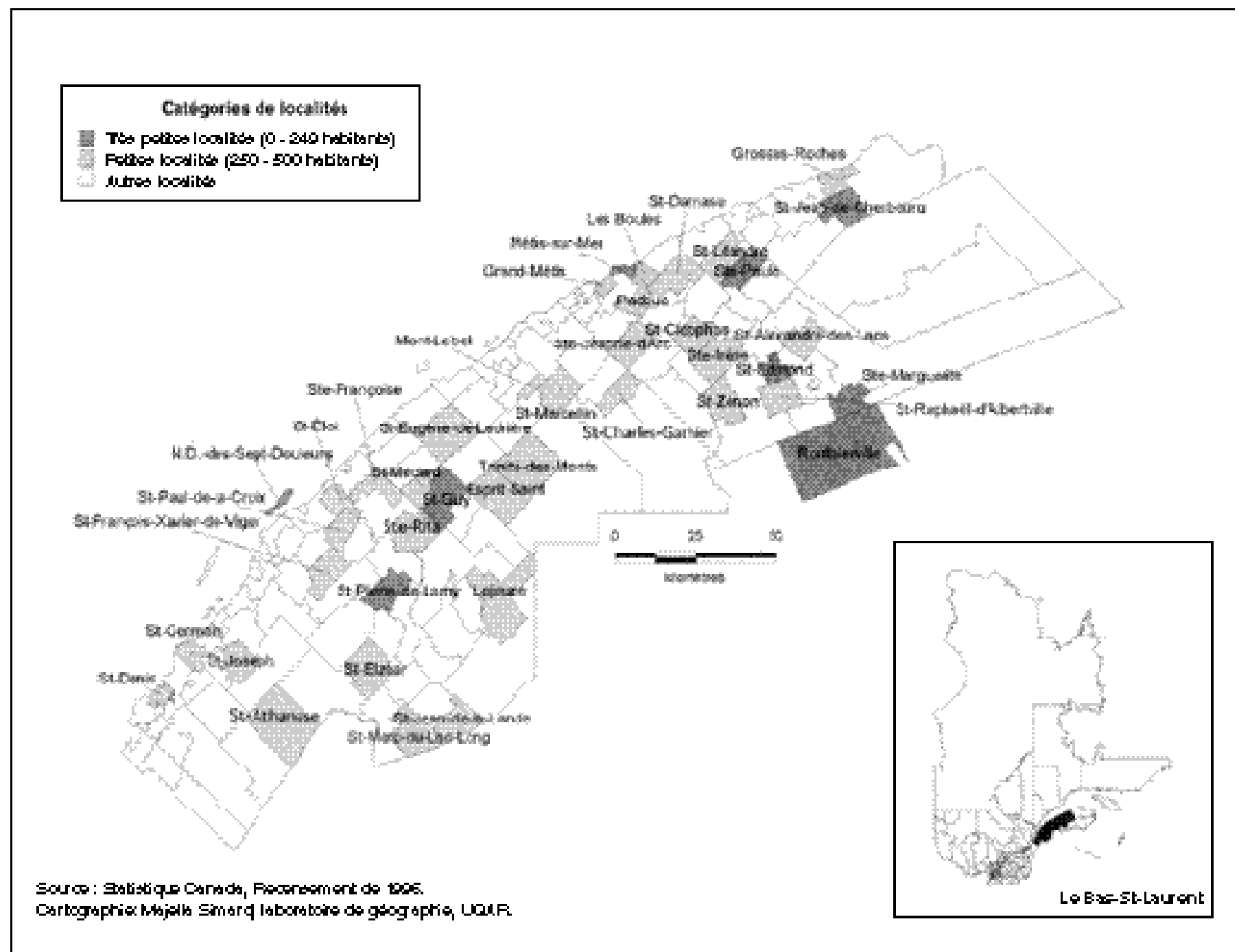

du segment constitué des petites localités, c'est-à-dire celles de 500 habitants et moins, qui présentent les problèmes socio-économiques les plus sérieux.

L'objet du présent article consiste, d'une part, à identifier les principaux malaises des petites localités rurales du Bas-St-Laurent au plan de la démographie. Une brève analyse, d'autre part, des caractéristiques de la structure occupationnelle, nous ouvrira quelques perspectives sur certaines réalités économiques et sociologiques du Bas-St-Laurent en général et sur celles des petites localités en particulier. Au nombre de 41, ces dernières abritaient, en 1996, 13783 individus, ce qui représente $6,7 \%$ de la population rurale régionale telle qu'elle est définie par Statistique Canada. Elles se localisent, pour la plupart, sur les terres ondulées des
Appalaches, bien que certaines longent également le littoral (carte 1).

L'approche que nous avons retenue pour illustrer ces différents malaises est celle des strates de taille démographique. Bien qu'imparfaite, cette méthode reflète de nombreux aspects de la réalité socio-économique du monde rural. Elle permet de mieux caractériser la nature des disparités, d'évaluer leur ampleur et de les localiser dans l'espace. La taille des localités influe sur l'étendue et la densité de l'écoumène, la quantité, la diversité et la qualité des services disponibles, la nature des occupations, la vie politico-relationnelle et la culture locale. Elle traduit aussi le rôle exercé par la localité dans la structure de peuplement. Par ailleurs, pour qu'il soit vraiment significatif, l'effet de taille 
doit être relativisé par d'autres variables parmi lesquelles les structures socio-spatiales, les éléments de localisation, la nature des économies locales et la vie fonctionnelle exercent un rôle d'avant-plan. Nous ferons régulièrement référence à ces différents éléments afin de mieux cerner les problèmes socioéconomiques de ces petits milieux dont la plupart sont en panne de développement.

Les problèmes des petites localités rurales du BasSt-Laurent sont d'autant plus menaçants qu'ils s'inscrivent dans une région déjà aux prises avec de sérieuses difficultés au plan socio-économique. Dans ce contexte, il nous apparaît essentiel de peindre, dans un premier temps, un tableau sommaire de l'état actuel des disparités et de voir brièvement en quoi la politique actuelle de développement local et régional du gouvernement du Québec s'avère inefficace pour les résorber.

\section{Persistance des disparités et développement régional}

Si le Bas-St-Laurent fait figure de chef de file dans les domaines des communications (Telus à Rimouski), du transport en commun (Bombardier à La Pocatière), de l'électronique (Premiertech à Rivière-du-Loup) et de l'océanographie (Institut des sciences de la mer à Rimouski), il n'en demeure pas moins que cette région, traditionnellement reconnue comme un territoire à problèmes, accuse de profondes disparités de développement par rapport au reste de la province. Le tableau 1 illustre bien l'ampleur des écarts qui apparaissent comme un paramètre déterminant des transformations de la société québécoise, que ce soit sur le plan de la croissance de la population, du taux d'activité, de la scolarité, de la structure occupationnelle et des niveaux de revenus.

Dans la foulée de ces données statistiques, il importe de rappeler les nombreuses pertes que la région a dû essuyer au cours des dix dernières années en raison de la restructuration des services des grandes sociétés d'État telles que Télé-Québec, Via Rail, RadioCanada, l'Office national du film et Postes Canada. De plus, la réorganisation du Mouvement Desjardins provoque de nombreuses craintes tant en milieu urbain qu'en milieu rural. L'abolition des fédérations régionales risque d'avoir des effets considérables sur l'économie rimouskoise, déjà affectée par les réductions de personnel dans la fonction publique québécoise. Du même souffle, la reconfiguration du
Si le Bas-St-Laurent fait figure de chef de file dans les domaines des communications (Telus à Rimouski), du transport en commun (Bombardier à La Pocatière), de l'électronique (Premiertech à Rivière-duLoup) et de l'océanographie (Institut des sciences de la mer à Rimouski), il n'en demeure pas moins que cette région, traditionnellement reconnue comme un territoire à problèmes, accuse de profondes disparités de développement par rapport au reste de la province.

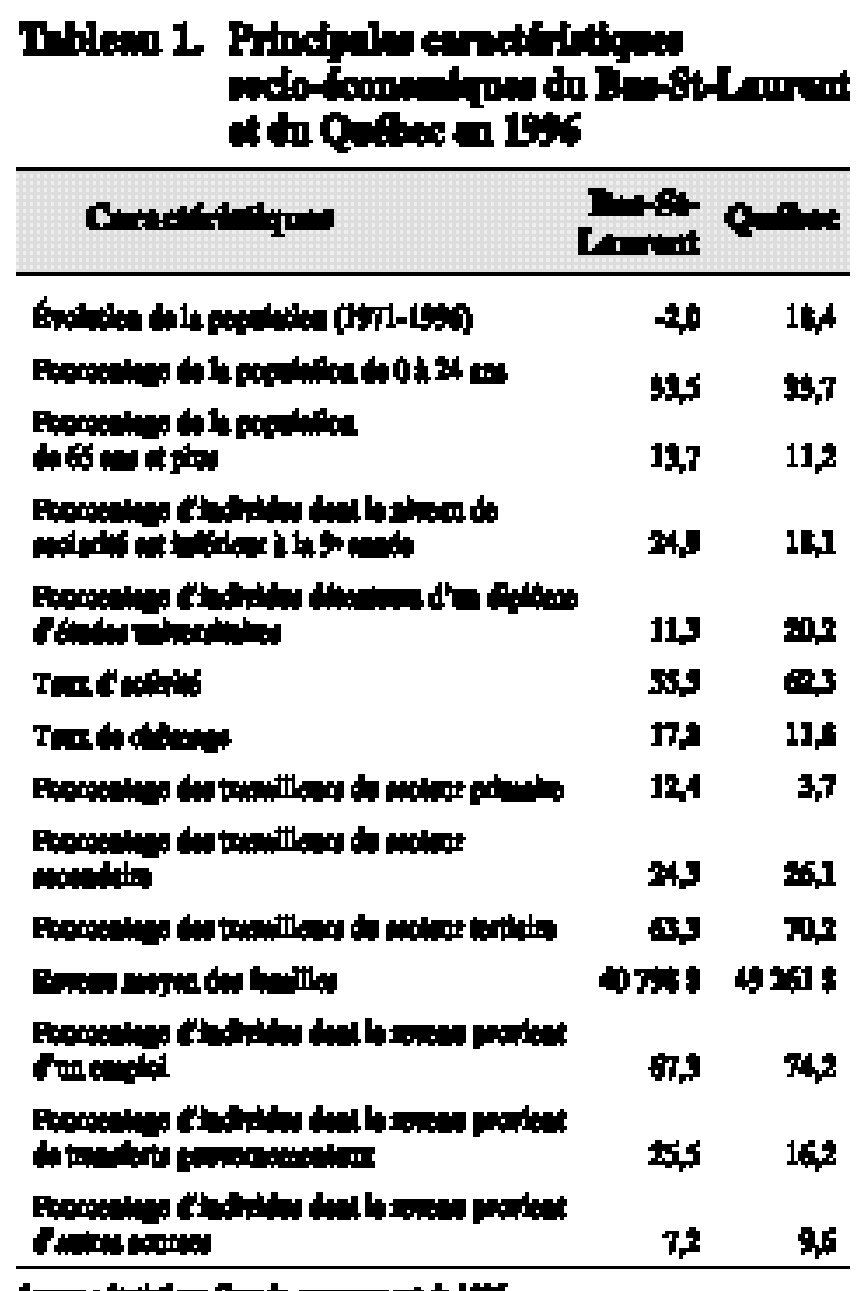

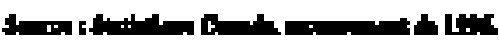


réseau des caisses peut être lourde de conséquences sur l'offre de services dans les petites localités rurales. Des rumeurs planent également sur la fermeture probable des sièges régionaux de Loto-Québec et de la Banque nationale du Canada, qui ont présentement pignon sur rue dans la capitale régionale. Enfin, la réforme municipale présentement en cours est loin de rassurer les élus des plus petites localités, qui craignent de perdre le faible poids politique dont ils disposent. Si la création de 400 emplois dans le domaine des nouvelles technologies de l'information et des communications (annoncée en grande pompe par le ministre des Finances du Québec en novembre dernier) est susceptible d'apporter un peu de sang neuf à l'économie régionale, il est peu probable que ces emplois parviennent à compenser les compressions draconiennes et les mesures radicales imposées par ce même gouvernement depuis la fin des années 1980, que ce soit dans les domaines de la santé, de l'éducation, de l'aménagement du territoire ou des services à la population en général. De plus, ces emplois peuvent difficilement faire contrepoids aux diverses caractéristiques structurelles qui font du Bas-St-Laurent une région mal intégrée sur le plan économique.

\section{Principaux malaises des petites localités rurales du Bas-St-Laurent}

\section{Effet de taille et disparités socio-économiques}

Puisque tout semble avoir été mis en œuvre pour assurer la rentabilité des régions dotées au départ des meilleurs avantages comparatifs, les espaces à population dispersée sont demeurés en marge des principaux progrès réalisés ailleurs. Cette forme de laisser-faire a notamment eu pour conséquence de favoriser le renforcement des disparités intrarégionales, particulièrement dans les milieux à très faible densité humaine.

Le tableau 2 fait ressortir quelques grandes tendances fort significatives ${ }^{4}$ en ce qui concerne certaines caractéristiques socio-économiques de ces milieux. Il s'en dégage deux niveaux différents de disparités socioéconomiques, d'une part, entre les petites localités rurales du Québec et celles des autres strates démographiques, et d'autre part, entre les petites localités rurales du Bas-St-Laurent et celles de la province. Ce même tableau met également en évidence l'existence de relations associatives entre la taille démographique des localités et certains indicateurs, notamment le revenu moyen $(0,42)$, le pourcentage des travailleurs du primaire $(-0,33)$ et le pourcentage d'individus n'ayant pas atteint le seuil de la $9^{\mathrm{e}}$ année $(-0,32)$ qui possèdent les coefficients de corrélation les plus élevés ${ }^{5}$. Nous constatons, en effet, que les disparités ont tendance à s'amplifier en fonction de la réduction de la taille démographique des localités. Les plus petites constituent le segment où les malaises socioéconomiques se manifestent avec une plus grande ampleur. Ces malaises sont d'autant plus inquiétants lorsque les entités en cause se localisent dans une région périphérique, en l'occurrence le Bas-StLaurent, déjà fragilisée par la nature de son économie et les différentes caractéristiques liées à sa structure de peuplement. La fragilité des petites localités rurales se reflète particulièrement sur le plan de la démographie. Cette dernière constitue une variable hautement significative de l'état de santé socio-économique d'un territoire. Elle résulte d'une grande variété de facteurs à la fois culturels, économiques, sociaux et même politiques. Elle synthétise de multiples transformations qui affectent le présent et sont déterminantes pour l'avenir. Elle exerce aussi un rôle considérable sur l'organisation socio-politique des localités. Enfin, elle reflète certaines caractéristiques liées à l'activité économique et à l'infrastructure de services. Bien que l'on retrouve des cas de décroissance dans toutes les catégories de localités, ils se présentent en plus grand nombre dans les plus petites, qui apparaissent comme les premières victimes du vaste mouvement de restructuration du tissu de peuplement rural. En effet, on remarque, à l'examen du tableau 2, que plus la taille des localités est petite, plus la propension au dépeuplement est élevée. Le taux d'évolution par strate de taille évolue du négatif au positif et en ordre de grandeur en fonction de l'augmentation de la taille de la localité. Celles de 500 habitants et moins constituent le seul groupe dont la population a décru $(-1,0 \%)$ entre 1991 et 1996. 


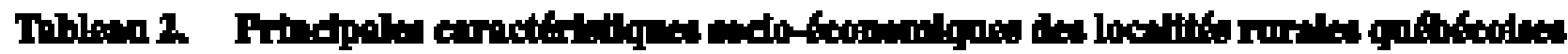

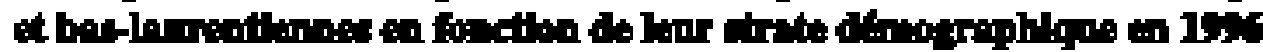

\begin{tabular}{|c|c|c|c|c|c|c|}
\hline \multirow[t]{2}{*}{ Thathatrin } & \multirow{2}{*}{ 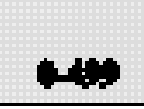 } & \multicolumn{5}{|c|}{ Thatomomith } \\
\hline & & 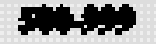 & 1 w1: & 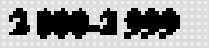 & 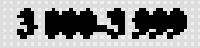 & $404 * 2$ \\
\hline \multicolumn{7}{|c|}{ 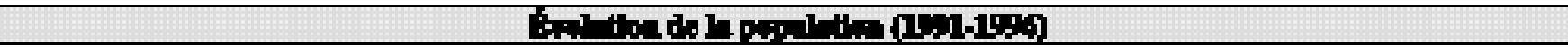 } \\
\hline 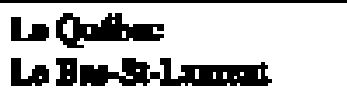 & $\begin{array}{l}-1,0 \\
-3,8\end{array}$ & $\begin{array}{r}1,7 \\
-1,6\end{array}$ & $\begin{array}{l}3 \mathbf{3} \\
0,0\end{array}$ & $\begin{array}{l}4,4 \\
0,4\end{array}$ & $\begin{array}{l}4,2 \\
0,4\end{array}$ & 4,4 \\
\hline \multicolumn{7}{|c|}{ 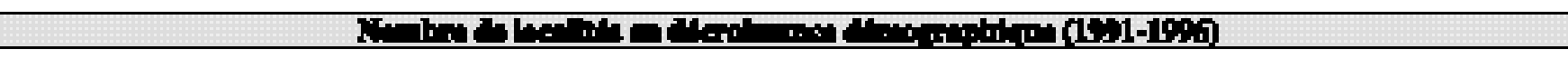 } \\
\hline 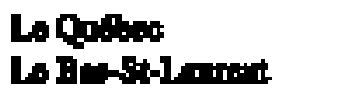 & $\begin{array}{r}187 \\
31\end{array}$ & $\begin{array}{r}187 \\
25\end{array}$ & $\begin{array}{r}111 \\
17\end{array}$ & 4 & $\mathbf{3}$ & 0 \\
\hline \multicolumn{7}{|c|}{ 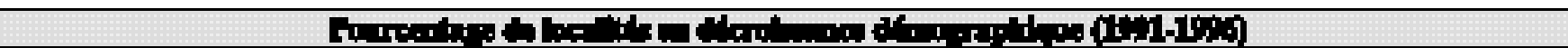 } \\
\hline 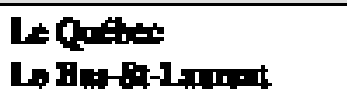 & 56,4 & $\begin{array}{l}45,3 \\
\mathbf{5}, 9\end{array}$ & $\begin{array}{l}52,6 \\
5,6,7\end{array}$ & $\mathbf{2 9 , 0}$ & $\begin{array}{l}27,0 \\
50,0\end{array}$ & $\begin{array}{r}10,5 \\
0\end{array}$ \\
\hline \multicolumn{7}{|c|}{ 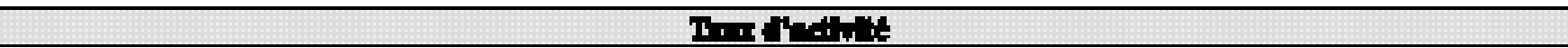 } \\
\hline 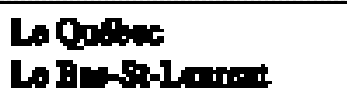 & $\begin{array}{l}55,2 \\
50,2\end{array}$ & $\begin{array}{l}36,8 \\
51,9\end{array}$ & $\begin{array}{l}5,0 \\
3,4\end{array}$ & $\begin{array}{l}612 \\
57,4\end{array}$ & $\begin{array}{l}61,7 \\
535\end{array}$ & $\begin{array}{l}63,5 \\
6,0\end{array}$ \\
\hline \multicolumn{7}{|c|}{ 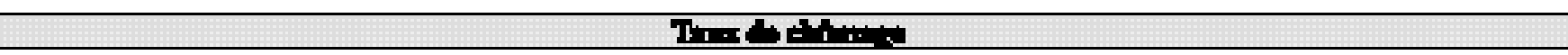 } \\
\hline 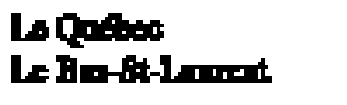 & $\frac{20,2}{26,9}$ & $\begin{array}{l}13,1 \\
25,4\end{array}$ & $\begin{array}{l}18,7 \\
128\end{array}$ & $\begin{array}{l}13,6 \\
15,4\end{array}$ & $\begin{array}{l}124 \\
13,9\end{array}$ & $\begin{array}{r}10,9 \\
2,9\end{array}$ \\
\hline \multicolumn{7}{|c|}{ 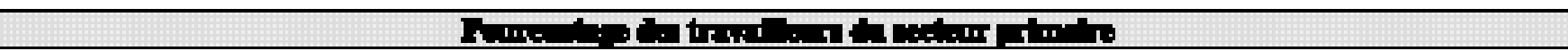 } \\
\hline 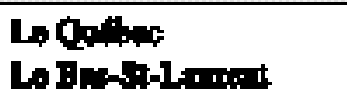 & $\begin{array}{l}20,4 \\
25,7\end{array}$ & $\begin{array}{l}1 \pm 0,0 \\
\mathbf{2 0 , 4}\end{array}$ & $\begin{array}{l}12,1 \\
15,0\end{array}$ & $\begin{array}{l}9,9 \\
7,2\end{array}$ & $\begin{array}{l}7,9 \\
9,7\end{array}$ & $\begin{array}{l}5,0 \\
2,7\end{array}$ \\
\hline \multicolumn{7}{|c|}{ 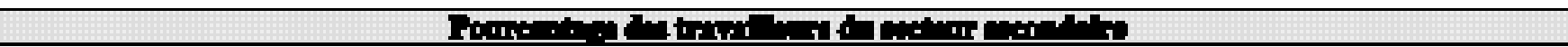 } \\
\hline 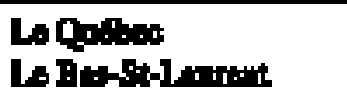 & 27,4 & $\begin{array}{l}\mathbf{3 2 0}, 0 \\
\mathbf{2 0 , 1}\end{array}$ & 34,1 & $\begin{array}{l}31,7 \\
24,6\end{array}$ & $\begin{array}{l}31,2 \\
23,3\end{array}$ & $\begin{array}{l}29,1 \\
18,3\end{array}$ \\
\hline \multicolumn{7}{|c|}{ 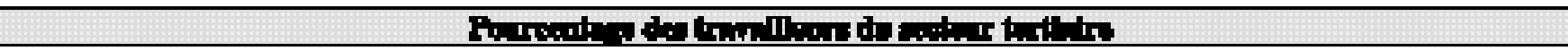 } \\
\hline 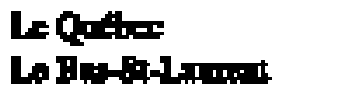 & $\begin{array}{l}52,2 \\
48,2\end{array}$ & $\begin{array}{l}50,0 \\
49,8\end{array}$ & $\begin{array}{l}\mathbf{5 5 , 9} \\
\mathbf{5 6 , 7}\end{array}$ & 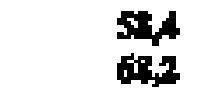 & 60,9 & $\begin{array}{l}6,9 \\
7,0,0\end{array}$ \\
\hline \multicolumn{7}{|c|}{ 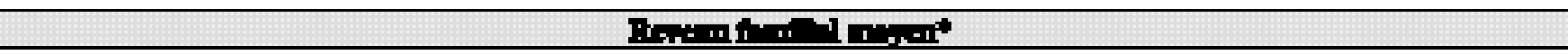 } \\
\hline 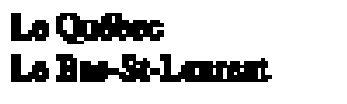 & 371238 & $\begin{array}{l}39404 \% \\
35594\end{array}$ & 41758 & $\begin{array}{l}44055 ! \\
42467 \%\end{array}$ & $\begin{array}{l}45762 \% \\
42067\end{array}$ & $\begin{array}{l}47535: \\
51 \mathrm{sm}:\end{array}$ \\
\hline \multicolumn{7}{|c|}{ 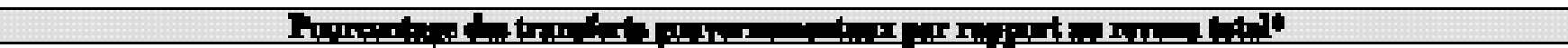 } \\
\hline 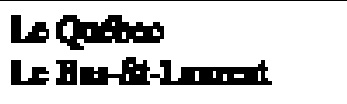 & 20,4 & $\begin{array}{l}\mathbf{2 6 , 1} \\
\mathbf{3 , 0}, 0\end{array}$ & $\begin{array}{l}20,5 \\
3,5\end{array}$ & $\begin{array}{l}20,3 \\
2,5,5\end{array}$ & $\begin{array}{l}18,1 \\
2,1\end{array}$ & $\begin{array}{l}17,1 \\
15,6\end{array}$ \\
\hline \multicolumn{7}{|c|}{ 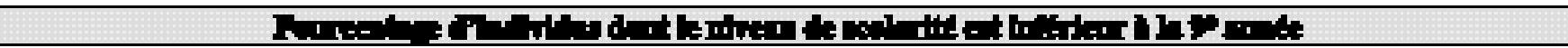 } \\
\hline 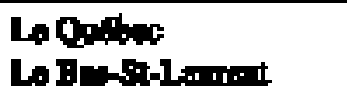 & $\begin{array}{l}3,1 \\
\mathbf{2 0 5}\end{array}$ & $\begin{array}{l}\mathbf{2 1 , 9} \\
\mathbf{2 3 , 3}\end{array}$ & $\begin{array}{l}19,6 \\
20,0\end{array}$ & $\begin{array}{l}17,7 \\
17,1\end{array}$ & $\begin{array}{l}16,0 \\
19,7\end{array}$ & $\begin{array}{r}14,1 \\
9,7\end{array}$ \\
\hline \multicolumn{7}{|c|}{ 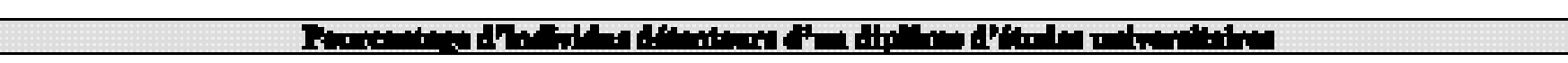 } \\
\hline 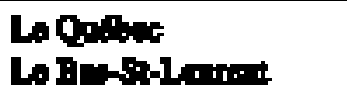 & $\begin{array}{l}2,2 \\
1,5\end{array}$ & $\begin{array}{l}2,2 \\
2,1\end{array}$ & $\begin{array}{l}2,7 \\
2,1\end{array}$ & 3,3 & $\begin{array}{l}3,3 \\
2,9\end{array}$ & $\begin{array}{l}39 \\
55\end{array}$ \\
\hline
\end{tabular}

4.

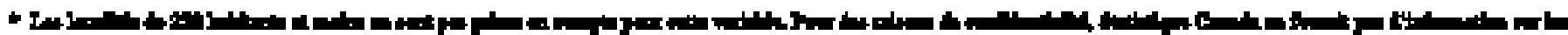

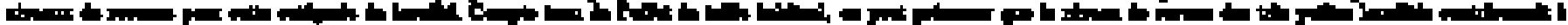

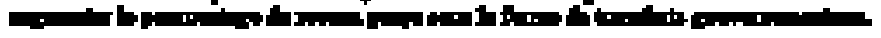


Le graphique 1 illustre bien l'aspect structurel du problème puisque les effectifs démographiques, dans cette portion de l'espace rural québécois, est en régression constante depuis les vingt-cinq dernières années. La décroissance de ce même segment est particulièrement significative dans le Bas-St-Laurent, où elle affecte les trois quarts des petites municipalités.

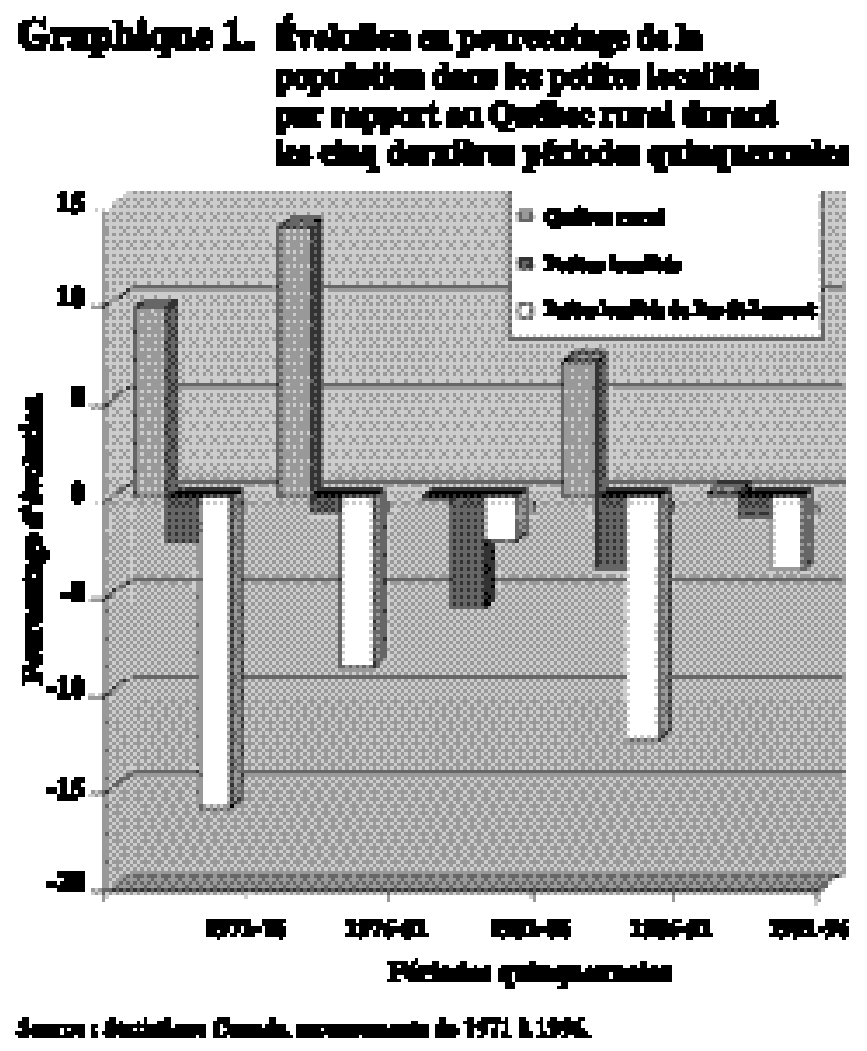

Les localités de 500 habitants et moins se distinguent par un faible taux d'activité et, par voie de conséquence, par un taux de chômage élevé. Ce dernier passe graduellement de $20,2 \%$ dans les municipalités de 0 à 499 habitants à $10,9 \%$ dans celles de 4000 à 4999 habitants. Il existe également une importante différenciation des structures d'emplois en fonction de la taille démographique des localités. Ainsi, les pourcentages des travailleurs du primaire sont quatre fois plus nombreux dans les petites localités que dans celles dont la population oscille entre 4000 et 4999 personnes.

Cet effet de taille s'observe particulièrement dans le Bas-St-Laurent, que d'aucuns qualifient régulièrement de «région-ressource» en raison d'un tissu économique largement dominé par l'exploitation des ressources naturelles. Dans la majorité des cas, les pourcentages élevés, que l'on observe dans les milieux faiblement peuplés, ne traduisent pas nécessairement une mise en valeur intensive des ressources locales. Ils reflètent plutôt la très grande fragilité de leur structure économique et le faible niveau de diversification de cette dernière. La relation entre la taille démographique des localités et le pourcentage des travailleurs du secondaire est moins évidente. Ce dernier fluctue considérablement d'une strate à l'autre. Ces fluctuations s'expliquent notamment par le fait qu'il existe peu de lieux de transformation et de mise en marché dans les petites localités alors qu'on les retrouve surtout en milieu urbain ou semi-urbain, ce qui amène pour plusieurs une forte dissociation entre le lieu de travail et de séjour ${ }^{6}$.

Bien que le secteur tertiaire soit le plus grand pourvoyeur d'emplois en milieu rural, il représente, selon les endroits, une réalité fort différente tant par la nature de ses activités, les volumes d'emplois, la quantité et la qualité des services offerts que par les revenus qu'ils créent. Pour bon nombre de municipalités, particulièrement dans les plus petites, les emplois que génère ce secteur d'activité sont occupés à l'extérieur de la localité de séjour. Généralement liés aux ressources naturelles et au tourisme, ils se caractérisent par leur saisonnalité, leur irrégularité et leur faible rémunération. S'il semble se dégager un lien causal entre la taille démographique des localités et le pourcentage de travailleurs du tertiaire, ce lien, pour qu'il soit significatif, est à mettre en relation avec d'autres variables, dont la localisation par rapport aux villes, le rôle des centres de services, la nature des activités économiques et la structure de l'économie régionale. D'après les statistiques du tableau 2 , les travailleurs du tertiaire ne représentent que $52,8 \%$ de la structure occupationnelle des petites localités comparativement à 65,9\% dans celles de 4000 à 4999 habitants. Il faut dire que la plupart de celles-ci exercent le rôle de petit centre de services dans leur environnement immédiat. Ces mêmes statistiques démontrent aussi que la relation entre la taille démographique des localités et le pourcentage des travailleurs du tertiaire est loin d'être parfaite. Cette imperfection se traduit notamment par un pourcentage plus élevé, pour cette catégorie de travailleurs, dans les localités de moins de 500 habitants que pour celles de 500 à 999 personnes. De plus, le Bas-St-Laurent affiche, dans quatre strates démographiques différentes, un pourcentage plus élevé de cette même variable que pour l'ensemble du Québec rural (c'est-à-dire dans les municipalités dont 
la population est inférieure à 5000 habitants). Ceci démontre bien la très grande diversité qui prévaut entre chaque catégorie de localités d'une part et la multiplicité des facteurs en présence de l'autre. Enfin, le pourcentage de travailleurs du tertiaire est aussi indicatif de l'importance de l'infrastructure de services à l'intérieur et à proximité de la localité. Dans les plus petites, cette infrastructure se résume, sauf exception, à peu de choses : la municipalité, l'église, l'école, le bureau de poste, le dépanneur et la caisse. À plusieurs endroits, certains de ces services sont sérieusement menacés ou ne sont carrément plus dispensés en raison de la faiblesse des effectifs démographiques à desservir et des coupures draconiennes exercées dans les budgets qui leur sont alloués. C'est notamment le cas à St-Guy, Trinité-des-Monts, Ste-Marguerite, St-Charles-Garnier et St-Jean-deCherbourg, où la dégringolade démographique des dernières années a été telle qu'elle met en danger la survie des services de première ligne. Le manque de clientèle associé aux mesures de rationalisation des commissions scolaires a forcé la fermeture de l'école à St-Marcellin, Ste-Marguerite, St-Cléophas, Notre-Dame-des-Sept-Douleurs, St-Alexandre-desLacs et St-Guy. Dans cette dernière localité, il ne reste plus aucun service commercial, ce qui représente un fait rarissime au Québec. À St-Jean-de-Cherbourg et à Ste-Marguerite, des infrastructures aussi symboliques que l'église ou le presbytère ont dû être démolies parce que les fabriques ne pouvaient plus assumer les coûts nécessaires à leur entretien. La fermeture récente de la coopérative de services a été un autre coup dur pour la petite localité de Ste-Marguerite maintenant dépourvue d'école, de station-service, de cantine, d'église et de caisse populaire. Cette municipalité est en voie de devenir un véritable hameau dortoir. Ses perspectives d'avenir apparaissent plutôt sombres.

Les petites localités disposent généralement de faibles revenus, ce qui nous amène, avec Dugas ${ }^{7}$, à établir un lien causal entre la taille démographique et les niveaux de revenus. $\mathrm{Si}$, pour l'ensemble du paysage rural québécois, cette relation semble parfaitement linéaire, cette linéarité souffre toutefois de nombreuses exceptions. À l'échelon du Québec rural, le revenu moyen passe graduellement de 32493 \$ pour les localités de moins de 500 habitants à $51822 \$$ pour celles comprises dans la strate des 4000 à 4999 habitants. Par ailleurs, chacune de ces strates comporte au moins 9 localités dont le revenu est supérieur à $52000 \$$. C'est le groupe des 1000 à 1999 habitants qui en comporte le plus (20 au total) alors que celui des 4000 à 4999 n'en renferme que 9 . On en dénombre 12 à l'échelon des petites localités. Il s'agit principalement de milieux miniers qui se retrouvent au Nord-du-Québec et en Abitibi. La progression est moins constante dans le Bas-St-Laurent puisque le revenu moyen des 2000 à 2999 habitants dépasse de $400 \$$ celui de la catégorie des 3000 à 3999 personnes. En dépit des variations moins fortes à l'intérieur de chaque strate, la relation entre la taille démographique des localités et leur niveau de revenu est incontestable.

La structure des revenus obéit également à l'effet de taille. Les revenus de transfert, qui constituent 17,1\% du revenu total dans les localités de 4000 à 4999 habitants, atteignent $29,4 \%$ dans celles de 500 personnes et moins. L'importance des revenus de transfert dans la composition du revenu total est symptomatique des nombreuses difficultés qui paralysent l'économie de ces petits milieux. Le Bas-St-Laurent se démarque par un pourcentage élevé des revenus de transfert dans toutes les catégories de localités mais plus particulièrement dans les plus petites.

Finalement, l'effet de taille se reflète également au niveau de la scolarisation. Les individus les plus scolarisés ont tendance à se concentrer dans les milieux plus urbanisés où l'on retrouve généralement les emplois plus spécialisés, les mieux payés et les plus stables. La population rurale du Bas-St-Laurent, dans la plupart des strates démographiques, comporte un pourcentage plus élevé d'individus n'ayant pas atteint le seuil de la $9^{\mathrm{e}}$ année et une proportion plus faible de personnes ayant obtenu un grade universitaire. Le phénomène se manifeste avec une plus grande acuité dans les localités de petite taille démographique où l'on retrouve les écarts les plus importants. De telles disparités sont le lot de nombreuses petites localités dont l'économie est fortement dépendante de l'exploitation des ressources, notamment de la forêt. Les emplois générés par celle-ci nécessitent généralement peu de spécialisation. Cette situation est d'autant plus difficile à redresser que les possibilités d'emplois pour les jeunes adultes sont généralement plus restreintes dans ce segment de l'espace rural. Cette sous-scolarisation est souvent le corollaire d'un autre phénomène, régulièrement évoqué dans les sciences humaines régionales, à savoir l'exode des jeunes provenant de ces milieux. Celui-ci, associé au vieillissement naturel de la population, est la cause d'un problème qui devient de 
plus en plus alarmant à l'échelle des petites localités : celui du dépeuplement.

\section{Évolution démographique des petites localités rurales du Bas-St-Laurent et redéploiement spatial de la population}

Dans le Bas-St-Laurent, plus qu'ailleurs, les petites et très petites localités (celles de 250 habitants et moins) ont été affectées par le processus de dépeuplement, particulièrement au cours des cinq dernières périodes quinquennales. Sauf pour la période de 1981 à 1986, la région se distingue par des pertes démographiques beaucoup plus considérables que pour l'ensemble des petites localités rurales du Québec (voir graphique 1). De tels écarts témoignent indubitablement de la déstructuration de tout un pan du territoire rural baslaurentien. Autre signe de fragilité : les petites localités ne cessent d'augmenter en nombre d'une période quinquennale à une autre. Ainsi, de 6 qu'elles étaient en 1961, leur nombre est passé à 28 en 1976, à 34 en 1986 et à 41 en 1996. Globalement, la population de ce segment, qui totalisait 35567 individus en 1951 (année où débute le processus de dépeuplement), a chuté à 13783 habitants en 1996 (dernière année du recensement canadien). Il s'agit d'une perte de 21784 personnes, ce qui représente une diminution de $61,2 \%$. La baisse de la natalité, une plus grande disponibilité des emplois à l'extérieur et une forte sollicitation de la vie urbaine constituent les principaux facteurs responsables de cette situation. Celle-ci cache cependant de profondes disparités qui traduisent les nombreux particularismes locaux liés au potentiel biophysique, à l'organisation de la structure de peuplement, à la force des sentiments d'appartenance, à l'économie, à la nature et à la qualité de l'infrastructure de services, à l'entrepreneurship, à la vie socioculturelle et aux orientations politiques.

La situation démographique des très petites municipalités apparaît particulièrement inquiétante (graphique 2). La saignée représente une perte de 4496 personnes en l'espace de 45 ans, soit une diminution de $75,4 \%$ comparativement à $56,3 \%$ pour l'ensemble des petites localités rurales bas-laurentiennes. Le phénomène est d'autant plus pernicieux lorsque ces dernières sont éloignées des villes et des principaux axes routiers. À St-Guy, Routhierville et St-Jeande-Cherbourg, trois municipalités situées à plus de 38 kilomètres d'un centre de services, la diminution de la population a été telle qu'elle menace l'existence
Dans le Bas-St-Laurent, plus qu'ailleurs, les petites et très petites localités ont été affectées par le processus de dépeuplement, particulièrement au cours des cinq dernières périodes quinquennales. Sauf pour la période de 1981 à 1986, la région se distingue par des pertes démographiques beaucoup plus considérables que pour l'ensemble des petites localités rurales du Québec.

même du village. Ces trois localités ont affiché respectivement des pertes démographiques de l'ordre de $85,8 \%, 85,2 \%$ et $82,8 \%$ durant la période de 1951 à 1996. Avec une diminution de 86,2 \%, Notre-Damedes-Sept-Douleurs représente un cas à part en raison de son caractère insulaire. Cette localité subit aussi de fortes variations saisonnières à cause de sa vocation touristique. Même si ces quatre localités sont aux prises avec de graves problèmes de déstructuration, on ne peut, en raison des constantes inflexions des courbes d'évolution et la mouvance de divers facteurs (sentiment d'appartenance, esprit de solidarité, implication sociale, valeurs personnelles, etc.), prédire leur fermeture à plus ou moins court terme. Par exemple, depuis quelques années, St-Jean-de-Cherbourg profite d'une légère croissance de sa population. Le nombre d'habitants de cette dernière est passé de 227 à 229 entre 1986 et 1991 . Au dernier recensement de 1996, cette même localité s'enrichissait de 10 nouveaux individus, portant sa population à 239 personnes. Il faut préciser ici qu'en regard des situations antérieures, le bilan démographique du recensement de 1996 a fait apparaître une nouvelle donne : 12 petites localités (dont trois très petites) ont enregistré une timide mais non moins significative augmentation de leur population. Il faut se reporter au recensement de 1956 pour observer un nombre aussi élevé de localités en croissance. Il s'agit d'un gain de 224 individus par rapport au recensement précédent. Un tel phénomène met en évidence deux faits majeurs. Premièrement, il témoigne du caractère imprévisible de l'évolution démographique des petites localités en raison des changements constants qui s'opèrent à l'intérieur de la trame de peuplement. Ensuite, il oblige à poser un sérieux bémol en ce qui concerne l'avenir de ces localités. Toutefois, il faut noter que cet 
accroissement ne contribue pas à compenser les pertes encourues depuis les quatre dernières décennies. Néanmoins, il a pour effet de maintenir un taux minimum de natalité suffisant pour assurer la survie de ces milieux fragiles. Par ailleurs, si rien n'est fait pour inverser la tendance démographique des très petites localités, plusieurs d'entre elles seront condamnées à la fermeture, à plus ou moins court terme.

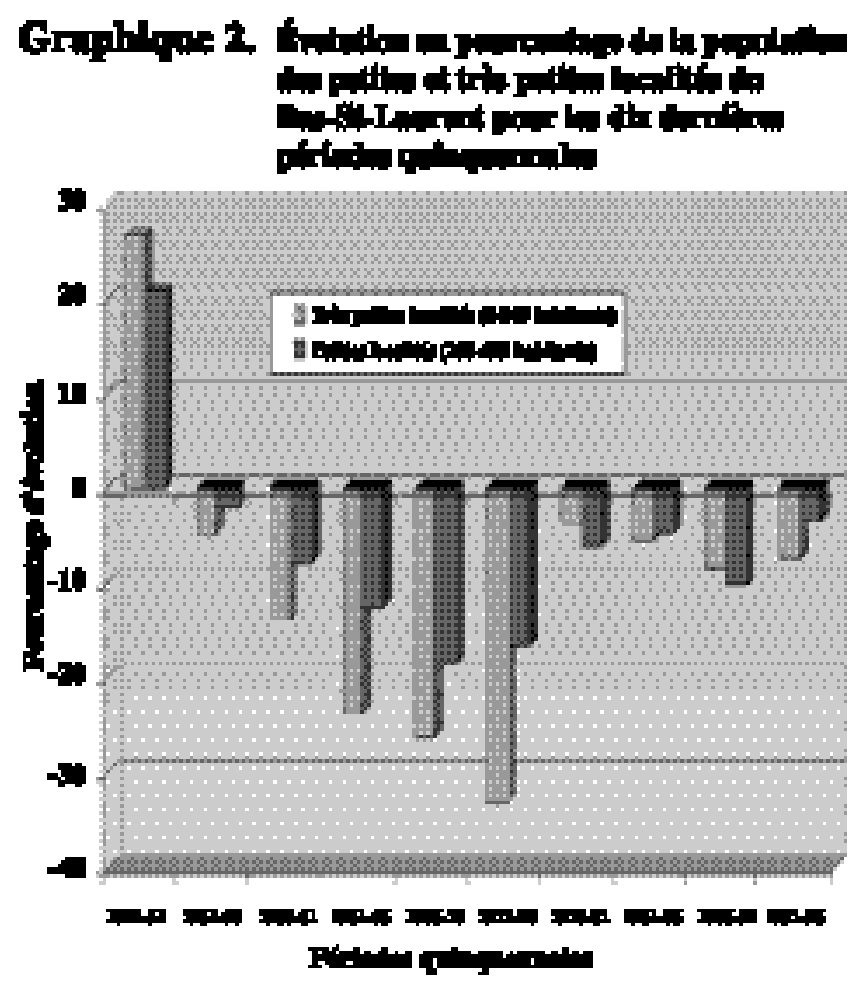

Sous-emploi et faiblesse des niveaux de revenu

Hormis quelques rares exceptions, les petites localités du Bas-St-Laurent sont paralysées par une économie fortement dépendante des ressources naturelles. Ces exceptions sont essentiellement de trois ordres. Elles concernent les milieux qui disposent d'un potentiel biophysique particulier (comme par exemple à St-Denis, où la qualité des sols permet l'exploitation d'une agriculture rentable), ceux qui abritent une entreprise de taille relativement importante (comme Les Boiseries du Bas-St-Laurent à Métis-sur-Mer) ou encore ceux qui profitent d'une situation géographique privilégiée (comme St-Damase ou St-Germain), qui se retrouvent au carrefour de plusieurs petites agglomérations.

En ce qui concerne le domaine agricole, de nombreux agriculteurs ont dû abandonner leur ferme en raison de leur incapacité de s'insérer dans une économie de type productivo-industriel. C'est ainsi que plusieurs hectares de terres agricoles sont retournés à leur état premier à Ste-Jeanne-d'Arc, St-Médard, Ste-Marguerite et St-Marc-du-Lac-Long. Là, l'agriculture n'est plus qu'un élément résiduel à connotation historique. Au mieux, on y pratique une agriculture d'autosubsistance. Faute d'une accessibilité suffisante à des marchés ou à des pôles de développement urbains, ces localités à économie fragile n'ont pas pu réaliser leur reconversion économique, d'où leur problème de dépopulation.

Si St-Médard (37,7 \%), Lejeune $(33,0 \%)$ et St-Zénon $(31,0 \%)$ concentrent une très forte proportion de travailleurs forestiers, ces localités ne disposent pas pour autant d'un potentiel exceptionnel. Dans ces milieux, la mise en valeur de deux ressources, l'agriculture et la forêt, s'est même avérée nécessaire pour assurer leur survie économique. La faiblesse des revenus locaux traduit bien la gravité de leurs problèmes économiques. Par conséquent, une relation associative se dégage entre la fragilité économique et la forte spécialisation dans le primaire forestier. En fait, à l'exception de St-Jean-de-la-Lande, peu de travailleurs forestiers exercent leur métier dans les secteurs les mieux rémunérés que sont le transport, le commerce et la transformation du bois. Étant donné que la forêt ne procure du travail que sur une courte période, de nombreux travailleurs doivent avoir recours aux prestations d'assurance-chômage afin de pallier le manque d'emploi. Cette situation explique le pourcentage élevé du revenu perçu sous la forme de transferts gouvernementaux dans ces localités. Ce dernier représente $65,0 \%$ du revenu total à St-Médard, $46,4 \%$ à St-Zénon et 38,6 \% à Lejeune.

Le revenu moyen des petites localités du Bas-St-Laurent en 1996 ne correspond qu'à $66 \%$ de celui de la province, qui se situe pour sa part à 49261 \$. Les localités à faible et très faible revenu ont tendance à se concentrer à l'est de la province. En effet, parmi les 20 petites localités les plus pauvres du Québec, on en retrouve 3 en Gaspésie et 6 dans le BasSt-Laurent. Il s'agit de Grosses-Roches, St-Zénon, St-Marcellin, St-Élzéar, St-Médard et Ste-Jeanned'Arc. La présence d'un grand nombre de localités pauvres témoigne indubitablement de la précarité économique qui affecte de vastes parties de l'espace rural de l'Est du Québec. Ces milieux souffrent de problèmes chroniques d'emploi. De plus, à l'exception 
de Grosses-Roches, ces localités se situent sur les hautes terres vallonnées des Appalaches loin des principaux pôles d'activité économique. Le revenu moyen de Ste-Jeanne-d'Arc ne correspond qu'à 38,6\% de celui de la province. Il découle non seulement du pourcentage élevé du revenu perçu sous forme de transferts gouvernementaux $(59,6 \%)$, mais aussi de la forte proportion de personnes âgées qui résident au sein de cette localité et qui ne retirent que le montant de base des prestations de la sécurité de la vieillesse.

Le revenu moyen des petites localités du Bas-St-Laurent en 1996 ne cor respond qu'à $66 \%$ de celui de la province, qui se situe pour sa part à 49261 \$. Les localités à faible et très faible revenu ont tendance à se concentrer à l'est de la province. En effet, parmi les 20 petites localités les plus pauvres du Québec, on en retrouve 3 en Gaspésie et 6 dans le Bas-St-Laurent.

\section{Conclusion}

$\mathrm{Au}$ terme de cette réflexion, une question centrale se pose, celle de l'inégalité des chances de tout un segment de l'espace rural, suivant une hiérarchie complexe - au même titre que d'autres traits de structure avec lesquels ils interagissent - des caractéristiques de leur localisation. Les nombreux problèmes auxquels les petites localités rurales du Bas-St-Laurent sont confrontées remettent sérieusement en cause leurs perspectives de développement. Cette catégorie de localités nécessite des actions structurantes de développement qui dépassent les capacités des seuls acteurs locaux. La persistance de ces difficultés témoigne de la nécessité d'élaborer de nouveaux mécanismes de développement. Les malaises de toute une partie de l'espace rural bas-laurentien et gaspésien comportent une importante dimension structurelle. Il faut donc, pour les corriger, élaborer des politiques ayant un contenu structurel orienté plus spécifiquement vers une meilleure répartition spatiale de la population et de la richesse.

Un autre type de développement est donc possible, une sorte de troisième voie qui consisterait à réconcilier l'éthique et l'économique en restaurant le prestige des valeurs humaines. Certes, l'élaboration d'un nouveau modèle fondé sur le principe de développement durable et plaçant l'humain au cœur des préoccupations n'est pas une mince affaire. Il suppose que nous rompions avec les pratiques et les «valeurs» des maîtres de notre monde et appelle des options radicales en matière de politique, de démocratie, de régulation économique et de distribution de la richesse collective.

\section{Notes et références}

1 Majella Simard est étudiant au doctorat en développement régional à l'Université du Québec à Rimouski.

2 Dans certaines localités, le taux de chômage dépasse facilement le cap des $50 \%$. C'est le cas notamment de l'Ascension-de-Patapédia $(68,0 \%)$, Rivière-à-Claude $(63,6 \%)$, La Martre $(56,0 \%)$, Ste-Madeleinede-la-Rivière-Madeleine (55,0 \%), Ste-Germaine-de-l'Anseaux-Gascons $(50,9 \%)$ et Listuguj $(50,0 \%)$, dont l'économie repose essentiellement sur l'industrie forestière et halieutique.

3 Ce mouvement est sans doute l'un des plus importants qu'ait connu la Gaspésie dans toute son histoire. Fier de ses 14000 membres, le regroupement, qui a pris naissance dans les MRC Côte-de-Gaspé et Pabok, a maintenant des ramifications jusque dans la région du Bas-St-Laurent, plus particulièrement dans les MRC de La Matapédia et des Basques. Au moment de sa formation, ses objectifs, on ne peut plus ambitieux, étaient essentiellement de deux ordres : intenter une poursuite de 15 millions de dollars en dommages et intérêts contre les gouvernements provincial et fédéral pour génocide économique, et obtenir un statut régional particulier (protectorat) afin que les Gaspésiens puissent déterminer eux-mêmes leurs stratégies de développement. Les leaders du mouvement, qui ont reçu l'appui de personnalités connues telles que l'avocat Guy Bertrand, le syndicaliste Michel Chartrand, l'auteur-compositeur-interpète Richard Desjardins et le comédien-chansonnier Jean Lapointe, se sont également engagés dans l'élaboration d'un plan de développement. Ce plan prévoit, entre autres choses, la mise sur pied de différents projets dont l'établissement de méga-porcheries, le développement du secteur bioalimentaire, la création d'un village-vacances à proximité du parc de la Gaspésie, la remise en service de la traverse Ste-Annedes-Monts - Sept-Îles, l'exploitation d'un gisement d'olivine, d'agate et de calcaire, l'ajustement des mesures fiscales provinciales et fédérales à la réalité régionale ainsi que les deuxième et troisième transformations de la crev ette et du crabe mousseux. Bien que le mouvement soit récemment re parti en campagne en raison du mécontentement de ses leaders à l'égard des gouvernements pour sortir la Gaspésie de son marasme, son action s'est quelque peu refroidie au cours des derniers mois. Ce refroidissement se reflète notamment par une participation de plus en plus mitigée des membres aux réunions. De plus, il semble régner au sein de ces derniers un climat de démobilisation 
peu propice à l'avancement des principaux dossiers pilotés par l'org anisme.

4 Nous insistons sur le fait qu'il s'agit de tendances et non de caractéristiques exclusives. De même, les indicateurs retenus, bien qu'ils contribuent à mettre en évidence la variabilité socio-économique de certaines localités, ne peuvent rendre compte de toute la diversité socio-spatiale du monde rural.

5 Pour les autres variables, soit le pourcentage des travailleurs du tertiaire, le taux d'activité, le taux de chômage, le pourcentage d'individus détenteurs d'un diplôme d'études universitaires, le pourcentage du revenu provenant de transferts gouvernementaux,l'évolution de la population entre 1991 et 1996 et le pourcentage des travailleurs du secondaire, ces coefficients sont respectivement 0,$26 ; 0,23 ;-0,22 ; 0,22 ;-0,13 ; 0,11$ et 0,05 .
6 Ce phénomène de migrations pendulaires (ou alternantes), c'est-à-dire la dissociation entre lieu de travail et lieu de séjour, n'est pas exclusif aux petites collectivités. Il s'observe dans toutes les catégories de localités. Cependant, les recherches de Clermont Dugas (1996) ont démontré que les plus petites, en raison de leur plus grande dépendance économique et sociale, disposent d'une plus grande proportion de migrants pendulaires comparativement à l'ensemble de l'espace habité.

7 DUGAS, Clermont (1999). Op. cit., note 4 ; DUGAS, Clermont (1990). «Les disparités de revenus dans l'Est du Québec», dans HÉTU, Bernard (dir.), L'Est du Québec: Études géographiques, Rimouski, Université du Québec à Rimouski, Module de géographie, p. 53-66. 